\title{
Les chants populaires grecs de la côte égéenne
}

What represents the popular song in Minor Asia, and especially near the Aegean coast?

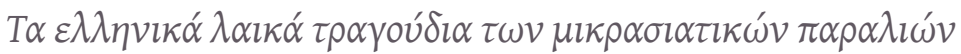

Katherine Nazloglou

\section{OpenEdition}

\section{Journals}

Édition électronique

URL : https://journals.openedition.org/ceb/1088

DOI : 10.4000/ceb.1088

ISSN : 2261-4184

Éditeur

INALCO

Édition imprimée

Date de publication : 9 janvier 2012

ISSN : 0290-7402

Référence électronique

Katherine Nazloglou, «Les chants populaires grecs de la côte égéenne », Cahiers balkaniques [En ligne], 40 | 2012, mis en ligne le 27 mai 2012, consulté le 06 juillet 2021. URL : http:// journals.openedition.org/ceb/1088; DOI : https://doi.org/10.4000/ceb.1088

Ce document a été généré automatiquement le 6 juillet 2021

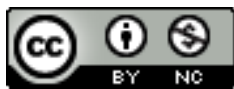

Cahiers balkaniques est mis à disposition selon les termes de la Licence Creative Commons Attribution - Pas d'Utilisation Commerciale 4.0 International. 


\title{
Les chants populaires grecs de la côte égéenne
}

\author{
What represents the popular song in Minor Asia, and especially near the Aegean \\ coast?

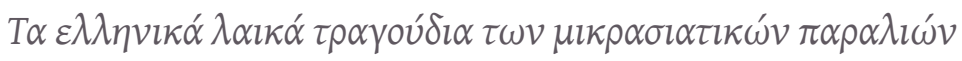

Katherine Nazloglou

1 Que représente la chanson populaire en Asie Mineure, et en particulier sur les rives de la mer Égée ? Le contexte du peuplement est cosmopolite, non seulement à Smyrne, mais dans les autres villes de la côte, tant les ports, même petits, en relation avec les îles proches et constituant le monde culturel et musical égéen, qu'à l'intérieur des terres. Depuis le XVIII ${ }^{\mathrm{e}}$ siècle, et plus encore avec les voies ferrées à la fin du XIX ${ }^{\mathrm{e}}$, échanges commerciaux et déplacements des hommes mettent en contact les centres urbains et les campagnes, les cités de la côte et de l'intérieur; au début du XX siècle, Salihli, Menemen, Pergame, Aïdin ne sont pas des centres isolés, même si la primauté de Smyrne l'emporte, mais des lieux de rencontres de diverses influences culturelles. Parmi elles, les influences musicales font de cet espace géographique un réel carrefour où les musiques byzantine, arabo-persane, ottomane, balkanique se mêlent à celles de la Grèce helladique et au rôle fondamental exercé par le karaghioze : influences sur les formes, les mélodies, les rythmes, mais aussi sur les thématiques des textes. La vie sociale, quotidienne, familiale, collective des Grecs du millet est marquée par la musique; c'est ce dont attestent les récits des voyageurs occidentaux, archéologues, hommes de lettres ou diplomates depuis le XVIII ${ }^{e}$ siècle, et Charles de Scherzer, Gaston Deschamps, Alfred Berl ou René Puaux entre autres, y relèvent la place de la chanson populaire.

2 Le compositeur et musicologue Louis-Albert Bourgault-Ducoudray publie à Paris, en 1876, au retour de son voyage en Orient, «Trente mélodies populaires de Grèce et d'Orient " puis l'helléniste Hubert Pernot et le journaliste-historien Georges-Frédérick Abbott marquent à partir de 1900 leur intérêt pour ces mélodies et les diffusent dans plusieurs publications. Dès 1903, les nombreux enregistrements réalisés à Smyrne, les 
tournées européennes de l'Estoudiantina de Smyrne, les lettres des voyageurs, la presse smyrniote, les cartes postales et les archives de recueils de chansons constituées par les Grecs dès les années 1920 sont les bases d'un intérêt transmis tant par la mémoire directe des réfugiés que par la recherche musicologique et littéraire. Les textes des chansons constituent en effet une source essentielle pour l'étude de la mémoire et des fonctionnements sociaux, des modes de vie, des mentalités des Micrasiates en tant qu'individus et organisations sociales particulières. En Grèce, le topos de la chanson "démotique » n'est pas urbain. En Ionie, il n'y a pas de réelle séparation culturelle entre les mondes urbain et rural, les implantations urbaines sont nombreuses, et d'autant plus sur et près de la côte. Smyrne est, certes, la plus importante, celle qui a donné son nom à une forme musicale et littéraire spécifique, mais des pratiques, des créations originales (par exemple, des rythmes, danses ou sujets dus à des environnements ou faits locaux particuliers) «s'observent ailleurs»: par exemple chansons de Phocée, Aïvali, Bournova, Aïdin, Pergame, Alatsata. Mais on trouve des transpositions, des variantes d'une ville, d'un village à l'autre; les appropriations, adaptations et transformations de rythmes, de modes comme de textes, les particularismes linguistiques, rendent parfois difficile l'établissement de la localisation originelle. De même entre Asie Mineure et Constantinople, et s'il ne faut pas, à notre avis, comme certaines analyses récentes l'affirment, englober dans une seule approche les deux domaines musicaux et géographiques dont les contextes politiques, socioéconomiques, culturels sont différents, il n'est pas question non plus de les dissocier voire de les opposer totalement, car les références mutuelles sont nombreuses.

3 À travers quelques exemples, que nous enseigne la chanson populaire ? ${ }^{1}$ Considérée comme une "mémoire des patries perdues ", elle est avant tout témoignage, expression de ces "patries». Elle est, originale ou adaptée, le produit de la petite bourgeoisie urbaine en pleine expansion de commerçants et artisans dans l'Ionie des années 1880-1922, c'est-à-dire la période correspondant à la Belle Époque, avec ses conventions sociales, morales. Elle a traversé, sans modification notable, par la voie orale essentiellement - jusqu'à l'arrivée du disque - les décennies et les générations. On la trouve, omniprésente, vivante, dans la société micrasiatique de 1922, dont les enfants en sont restés témoins et transmetteurs jusque dans les années 1970, quand la transmission orale se tarit et doit devenir mémoire écrite - ou chantée.

\section{L'expulsion}

Les Grecs d'Ionie, sujets de l'Empire ottoman, évoluent dans le contexte politique intérieur et international complexe de la période des relations internationales menant à la Grande Guerre. Victimes des relations particulières qui se développent entre le royaume de Grèce et l'Empire entre autres, ils sont objets d'expulsions, exactions; le massacre de Phocée, en 1913, en témoigne avant même le conflit mondial et sa conséquence, la guerre d'Asie Mineure dont le déroulement et l'issue signent la tragédie et la fin de l'hellénisme. Cependant, paradoxalement, peu de chansons font référence à ces persécutions et massacres.

Des témoins, tels Georges Metsolis et Nicolas Horbos ${ }^{2}$ ont, à partir de leur mémoire et de carnets familiaux constitués avant et après 1922, retranscrit les chansons de leur enfance. La plupart des "chansons de Phocée" qu'ils rapportent se retrouvent cependant, avec les mêmes textes ou avec des variantes ailleurs sur la côte égéenne et 
en Ionie. Quelques «chansons de la maison abandonnée » sont plus spécifiques des expulsions subies, ainsi :

Ma pauvre maisonnette! Les soins de tant d'années,

les joies de tant d'années l'avaient si bien parée

depuis ses fondations jusque sur le toit,

navire de la vie, armé pour nous porter.

Chargée de rêves, d'espoirs et de bonheurs,

de patience, de volonté, de nos désirs.

Toute une existence! Combien de fois

n'y a-t-on dressé la table nuptiale.

Maintenant muette, déserte et verrouillée,

la poussière et l'araignée vont la couvrir.

Et qu'importe la clef que pourtant j'ai gardée?

Car même sans clef, la porte, ils vont l'ouvrir. ${ }^{3}$

Mais un jour on la retrouvera,

mais un jour à nouveau nôtre tout sera ${ }^{4}$.

Refrain d'un espoir malgré tout, que l'on retrouve dans maintes chansons d'Asie Mineure et de Constantinople, et généralisé surtout après 1922. Mais dès avant 1914, une chanson de Pergame fait aussi référence au contexte politique :

PERGAMOS

Pergamos, oh ! belle Pergamos, nous partions et sur toi nous pleurions,

nous pleurions, mais ne pouvions oublier

la vie si belle que nous avions perdue

Dans le jardin et le verger qui étaient miens

je t'ai donné tout mon amour,

et quand j'ai eu vingt ans, mon aimé,

je t'ai fait le don de moi.

Pergamos, aman, aman, Pergamos,

nous partions et sur toi nous pleurions.

Nous pleurions sur nous tous, désormais si pitoyables

et nos larmes ternissaient la mer.

7 qui devient à partir de 1923, dans sa variante la plus connue, expression de la mémoire collective en intégrant dans le même souvenir douloureux tous les lieux marqués par la Catastrophe :

Pergame dont toujours nous parlons....

Aman aman Aïvali, Pergame et Aïvali

Aïvali, mon coeur a mal à toi,

Smyrne et Boutza

et Bourtza, Kordélio et Karatassi,...5

Aman aman, Phocéenne, mon coeur, Phocée, a mal à toi.

$8 \mathrm{Ph}$. Falbos, principal témoin des traditions populaires phocéennes, rapporte par ailleurs comme étant «de Phocée» une "chanson de septembre», quand les oiseaux repartent :

Un petit oiseau s'amuse

Et chante petit chanteur

Et feuille petite feuille

Loin de l'arbre en emporte l'écho ${ }^{6}$.

9 Le conte est un domaine très particulier du patrimoine populaire, et l'un des plus anciens. Falbos ${ }^{7}$ donne comme "phocéen » celui qui a pour héros le prince Fiorentino, sa mémoire ayant retenu le conte et les chansons du conte de son enfance; il a pu le retranscrire en grec à partir de ses souvenirs et, trouvé par hasard, d'un texte en 
français d'E. Legrand qui avait publié quatre contes en $1884^{8}$. Mais on en connaît d'autres versions puisqu'on le trouve à Zakinthos, dans le Péloponnèse, dans certaines îles (Crète) sous d'autres titres et noms du prince. Si la langue, certaines situations décrites sont incontestablement influencées par le contexte local, l'origine et le thème du récit ne sont pas micrasiatiques et encore moins phocéens; héritier d'une tradition orale de plusieurs siècles, ce conte, comme de nombreux autres, est originaire d'Italie et a traversé les mers au cours des évolutions historiques en se transformant, mais en perdurant. De plus, ici, il est adapté à la société urbaine phocéenne et plus largement ionienne. Le trait le plus significatif rapporté par Falbos est que la fin du conte était si célèbre à Phocée qu'elle était non seulement chantée, mais aussi dansée, même par les petites filles dans les quartiers populaires. La génération née entre 1910 et 1925 est la dernière à avoir conservé la mémoire orale directe des chansons chantées aux enfants par leurs mères, grands-mères, tantes ou apprises à l'école, pour les fêtes tant religieuses que profanes.

10 Comme Phocée, les ports et l'Asie Mineure égéenne sont des lieux d'exil. Un creuset de thèmes communs enrichit la chanson populaire et donne quelques-uns des textes les plus originaux et les plus représentatifs d'une société marquée par l'éloignement et l'exil. Exil de la jeune fille qui quitte son foyer et sa « petite patrie » pour une autre :

D'UN PAYS ETRANGER ${ }^{9}$

D'un pays étranger et lointain

Une demoiselle est venue, âgée de dix-huit ans.

Elle n'apparaît pas sur le seuil et jamais dans la rue

Ni même à sa fenêtre...

Exil du marin qui s'en va sur les mers proches ou de plus en plus lointaines, ainsi cette chanson particulièrement sombre d'Aïvali.

LE MARIN

Le marin est malade, mais à la proue du navire, Sans mère pour le voir ni père pour le soigner Ni frère ni soeur ne le prennent en pitié.

Le plaignent seulement le capitaine et le patron.

- Debout, marin, debout mon vaillant gars,

- Va contre le grain et conduis-nous au port.

- Moi je dis n'en pouvoir plus et vous criez « debout ».

Tenez-moi, je me lève, faites-moi donc asseoir, Donnez-moi la carte et le compas d'argent, Je vais contrer le grain, nous gagnerons un port. Vous voyez cette crête juste après le cap? Là nous jetterons l'ancre, dans le golfe profond. Les marins trouveront de l'eau et le cuistot du bois. Quant au petit mousse il y creusera ma tombe. Pas sur le sommet, ni même sur la pente, Juste au bord de la plage, dans les petits galets. $A u$ côté droit, qu'il laisse une échancrure, Que le soleil y entre le matin, les oiseaux à midi, J'aurai les caresses de la mer, les baisers de la vague Et le chant des marins.

Amis, quand vous irez à mon village voir ma bonne mère, Taisez la maladie, aussi que je suis mort, Mais dites que j'ai pris femme loin là-bas à l'étranger. Carj'ai pris la tombe pour marâtre, pour femme la noire glaise.

11 Vont s'y ajouter les thèmes de l'exil des émigrés, de plus en plus de Micrasiates partant pour l'Amérique ou l'Australie, à partir de 1909 de ceux qui refusent d'effectuer le 
service militaire, devenu obligatoire, dans l'armée ottomane; et à partir de 1912 de ceux qui s'engagent dans l'armée grecque durant les guerres balkaniques puis la Grande Guerre et celle de 1919-1922. Cependant, ces chansons "guerrières " ne sont pas originales, elles empruntent surtout aux domaines littéraire, rythmique et mélodique proprement grecs et en particulier klephtique. Sur des textes grecs, les références à Constantinople, à sa " prise » et à l'imaginaire micrasiatique qui s'y réfère, les termes techniques nés de ces guerres modernes, les toponymes grecs et balkaniques cités, souvent transformés ou mal localisés, car inconnus auparavant montrent qu'elles appartiennent à l'aire micrasiatique. Dans la plupart des cas, les chansons sont donc populaires musicalement parlant seulement.

\section{La chanson « narrative »}

12 Un des registres les plus riches de cette chanson populaire ancrée dans l'histoire de l'Ionie reste la chanson considérée comme "narrative », en particulier celle qui trouve sa source d'inspiration dans des faits héroïques, historiques, réels ou revisités par la mémoire collective et transformés par une lignée de transmission plus ou moins longue. Ainsi, une chanson d'Aïvali à propos d'événements y ayant eu lieu en 1821, comme en témoigne le Français Claude-Denis Raffenel qui rapporte le chiffre de 1650 tués ${ }^{10}$ :

\section{MOSCHONISSIA}

Trois petits oiseaux sur un arbre très haut,

L'un regarde Aïvali et l'autre Samothrace,

Le troisième quant à lui, regarde les Moschonissia.

- Tiens bon, cher Aivvali, et toi, ma Samothrace.

- Ils ne sont pas mille et deux mille, ils ne sont pas cinq ou dix Mais des mille et des cents que

nul ne peut compter.

Variante la plus commue :

... L'une pleure Aïvali et l'autre Moschessi (sic)

De toutes la plus jeune pleure Moschonissi.

Cette chanson, connue et attestée par des transcriptions depuis les années 1870 avec de nombreuses variantes jusqu'en Thrace, transmet, par-delà des faits historiques anciens que la mémoire a oubliés, la souffrance de la destruction d'Aïvali (laquelle à partir de 1923 , se confondra avec celle des persécutions et destructions ultérieures ${ }^{11}$.

\section{La chanson d'amour}

Le corpus le plus abondant est constitué par les chansons d'amour, qui font partie d'un ensemble plus vaste que nous pouvons qualifier de sociétal, où, à côté des chansons d'amour, voire érotiques, sont évoquées les relations familiales, les pratiques, même les plus humbles, de la vie quotidienne, les fêtes, les relations entre les différentes catégories de la population, entre les générations... À l'étonnement de voyageurs tels Ducoudray, Scherzer, c'est une société de la joie de vivre, de l'harmonie, du bonheur, ce dont rendent compte les témoignages tant littéraires que mémoriels dans l'« après » 1922. Cependant, si les chansons populaires nous parlent, certes, d'une réelle douceur de vivre, les plaintes, les lamentations, le désespoir y sont très présents. Le monde dépeint n'est pas uniquement celui d'un idéal de vie imaginé, souhaité, il est celui des réalités quotidiennes, des joies, des plaisirs, mais aussi des déceptions, des drames, du 
fatalisme dans lesquels évolue cette société. S'en dégage un immense amour pour la terre ionienne, ses paysages, ses lieux de vie, Smyrne évidemment, mais aussi toutes les "petites patries», sans distinction d'importance économique, ou de renommée. Bonheurs et malheurs privés, individuels et collectifs prennent place dans le cadre imposé par le millet, où l'on vit entre soi dans des quartiers spécifiques et hiérarchisés, que les Grecs représentent une population minoritaire ou fortement majoritaire parmi les autres, Turcs, Arméniens, Juifs, Levantins et Balkaniques de diverses origines.

Dans la chanson d'amour, les conventions d'une société fermée, tant urbaine que rurale, séparent nettement les hommes et les femmes. La femme dévoyée, «pas comme il faut ", est stigmatisée. Ainsi, Elli qui scandalisa la société smyrniote au début du XX $\mathrm{X}^{\mathrm{e}}$ siècle pour avoir trompé son mari avec un "Commissaire », Turc qui plus est, et devint célèbre, une chanson l'ayant, avec des variantes ultérieures, immortalisée :

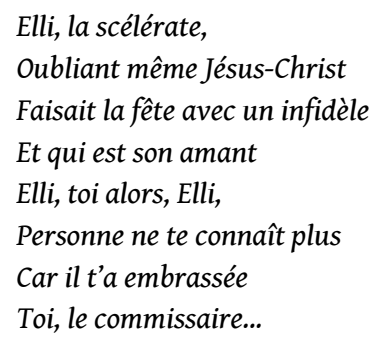

16 Cependant, on note à travers la chanson populaire une évolution considérable de la condition féminine durant les deux décennies précédant la "Catastrophe »; et dans la région de Smyrne plus qu'ailleurs, le grand port recevant davantage les innovations dues aux contacts avec l'extérieur. Traditionnellement, la femme joue un rôle fondamental dans l'ordre social, familial : fille, épouse, mère, veuve, sa place est fixée, ordonnée, codifiée. Les témoignages, tant des Micrasiates que de Grecs, d'Européens ne manquent pas pour vanter la beauté, l'allure, les appâts, devenus mythiques, des Smyrniotes (mais la société constantinopolitaine - jalouse? - leur reproche une « légèreté » qui en serait le corollaire); photos, cartes postales colorées ou non, les publicités commerciales des sociétés et magasins d'importation de tissus, vêtements, accessoires, tout ce qui fait la mode et la modernité d'Europe occidentale les exposent partout et montrent leur importance sociale et économique, quelle que soit par ailleurs la couche sociale, la famille, le quartier auxquels elles appartiennent. Gaston Deschamps, déjà en $1890^{12}$, bien que souvent critique à l'égard des Micrasiates, décrit les femmes comme belles (il n'a pas vu de «laides»!), est enthousiasmé par leur "profil Ionien", constate l'omniprésence de la musique dans les rues, et pas uniquement à Smyrne. Les cartes postales, en particulier, nous montrent les lieux de vie dans lesquels elles évoluent. Si les "kentra » (tavernes où l'on joue de la musique) restent presque exclusivement réservés aux hommes, à partir de 1900 on voit de plus en plus de femmes les accompagner au "Sporting Club», au «Louma Park», au café "Éden, au "Corso", chez Athanassoulas à Korkayali, au "Café-Paris", joyaux de l'architecture de Smyrne, dont certains sont situés sur les "Quais», et où l'on joue diverses musiques appréciées en Asie Mineure: "variété » occidentale, chansons des « revues » athéniennes, chanson italienne ou sa forme grecque, la « cantada ». Un chant fait référence au «Jardin des Fleurs », célèbre « kentro » situé dans un parc du quartier populaire de Stavros à Smyrne ${ }^{13}$.

JE T'AI VUE

Je t'ai vue au Jardin des Fleurs, j'en ai perdu l'esprit

Et n'ai pu retrouver la porte de ma demeure.. 
Les « chansons d'Asie Mineure » (les Micrasiatika) sont jouées dans les cafés-aman et à l'occasion des fêtes qui rythment la vie sociale, très largement organisée à l'extérieur, dans la rue, sur les places devant les églises, devant la maison. Elles n'évoquent que rarement (surtout en cas de crise comme nous l'avons évoqué précédemment) la " peur du Turc » et, si la vie l'intérieur des foyers est ordonnée, hiérarchisée avec ses principes et ses rites, les Micrasiates vivent fenêtres et portes ouvertes, les enfants jouent dehors, des pièces de l'habitation on entend crier les vendeurs de rues, les artisans ambulants, mais on reste "entre soi ", dans la protection apportée par l'organisation du millet ${ }^{14}$. Naissances, baptêmes, mariages, enterrements, deuils, fêtes du nom, de la paroisse, du saint patron, pèlerinages sont des occasions de sorties ou de pratique de «maison ouverte " aux parents, amis, voisins, de rencontres auxquelles toutes les générations et catégories sociales participent entre autres par la chanson. Chacune veut répondre aux particularismes de la "petite patrie " qu'elle exprime, mais ici aussi on note que les moeurs, les liens sociaux et familiaux, les rituels présentent de très fortes analogies tout le long de la côte égéenne. Elles décrivent l'environnement, la géographie, les lieux de vie, la maison la pièce à vivre et la cuisine où évoluent les femmes, les cathédrales des villes et leurs églises, les chapelles de campagne.

Les jardins sont un thème d'inspiration favori montrant l'importance de la nature, le rapport que les Micrasiates entretiennent avec elle, l'amour qu'ils portent à leur terre et qui explique ce que les témoignages, la poésie, la littérature et les chansons postérieures à 1922 transmettront de la mémoire des inoubliables "patries perdues ». Les chansons délivrent un lexique remarquable de fleurs, arbres et fruits (souvent oubliés, car inexistants en Grèce) qui permet souvent à l'auteur et à l'interprète de qualifier à l'infini tous les appâts de la bien-aimée avec des comparaisons très poétiques (le plus souvent sur le rythme du ballos). Un chant de Cesme ${ }^{15}$ égrène ainsi sur quarante vers les qualités d'une jeune fille, du sommet de la tête aux ongles des pieds avec autant de noms de fleurs et fruits. Ce principe se retrouve partout, mais les termes peuvent changer selon les lieux, mêlant grec ancien, mots locaux, voire turcs retranscrits. La poésie devient alors allégorique. Parfois ce sont les refrains qui permettent de localiser précisément la chanson ou sa variante, ainsi :

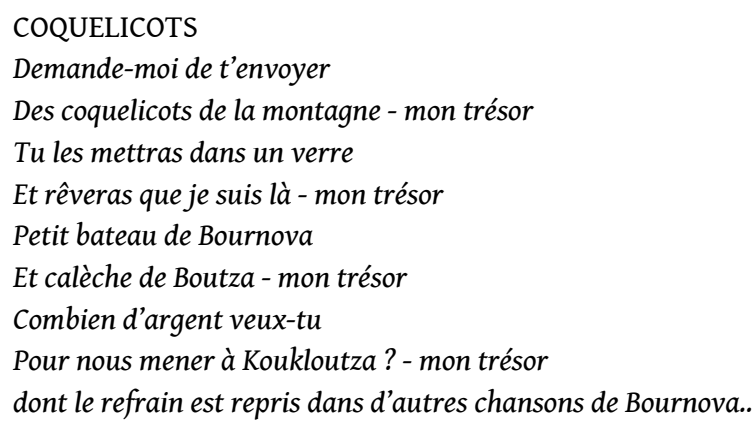

Dans la chanson d'amour, c'est traditionnellement l'homme qui adresse sa flamme à la jeune fille qui est strictement élevée, placée sous l'autorité de son père. Cependant des évolutions apparaissent, les chansons parlent ouvertement d'amours contrariées, de déceptions du jeune homme, d'amours interdites, cachées, où la parole est donnée à la femme, à la jeune fille qui refuse un parti, fait valoir ses préférences, sa volonté.

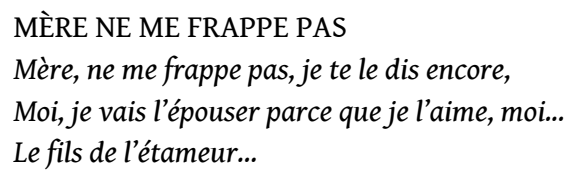


Ici le chant est celui de la jeune fille qui veut épouser un garçon de condition inférieure à la sienne, ce que le code familial récuse, mais le nombre de chants sur ce thème, est tel qu'il confirme des évolutions sociales notables.

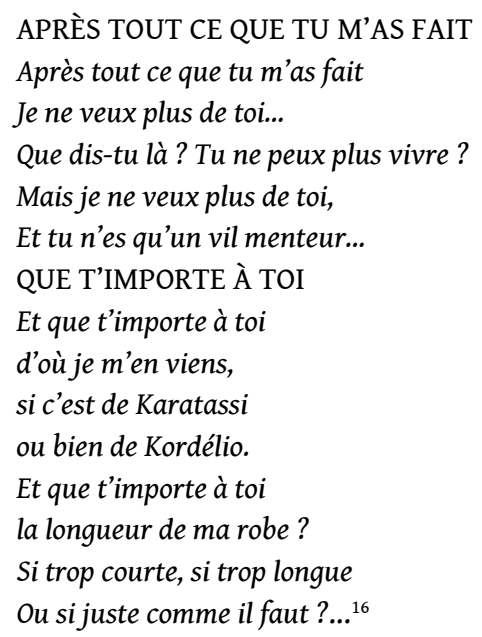

21 Et quand l'homme parle, c'est aussi pour se plaindre de l'indifférence de l'être aimé et prendre l'attitude du suppliant :

\section{GEORGETTE}

Moi, je voulais t'aimer ma Georgette,

que personne, oh! non, personne ne le sache,

mais les tiens l'ont appris, ma Georgette,

ils l'ont appris - oui, l'ont appris -, les étrangers aussi.

Youla, youla, youla, viens et me prends,

ouvre tes bras tous grands, mets-moi dedans,

viens, youla, youla, youla.

Le jasmin sur ton seuil, ma Georgette à moi,

est en fleurs, il est en fleurs et va grandir.

Ton corps d'ange, ma Georgette, ma Georgette à moi,

Dans mes bras, oui dans mes bras va tomber.

Viens et me prends, youla, youla, youla,

toi qui as embrasé mon petit cœur,

viens, youla, youla, youla.

Cueille les fleurs du jasmin, ma Georgette à moi,

et je les enfilerai, oui je les enfilerai,

vends-moi ton amour, ma Georgette, ma Georgette à moi,

et je l'achèterai, oui, je te l'achèterai.

Les amours contrariées ne sont plus obligatoirement cachées, en témoigne un chant, précisément daté, qui fait expressément référence à l'entrée de l'armée grecque dans Smyrne en 1919.

PETITE SMYRNIOTE

Ma douce petite Smyrniote

Avec tes si grands yeux

Mon coeur infortuné

Tu l'as déchiqueté.

Viens, unissons-nous tous deux

Ma belle amie

Comme Smyrne vient de s'unir

Avec la Grèce.

Dans un palais bien pauvre,

Viens, unissons-nous tous deux, 
Puisque dans notre Smyrne

L'evzone y est entré

Le rapport à la mer imprègne la vie quotidienne des Grecs le long de la côte égéenne. La chanson populaire exprime toute la gamme de ces liens et des sentiments que le Micrasiate éprouve à son égard, Associée à l'aimée, à la " petite patrie ", aux divers métiers qui font vivre une partie de ses riverains, aux cultes de saints, chapelles et rites religieux qu'elle suscite comme aux drames qu'elle engendre, à la mort qu'elle provoque, la mer symbolise l'Ionie, elle est la mère tout comme la terre féconde, et porte, au-delà des thèmes de l'exil. On la vénère, on la craint et la maudit, mais elle reste la nécessaire présence qui accompagne nombre d'actes quotidiens ou exceptionnels.

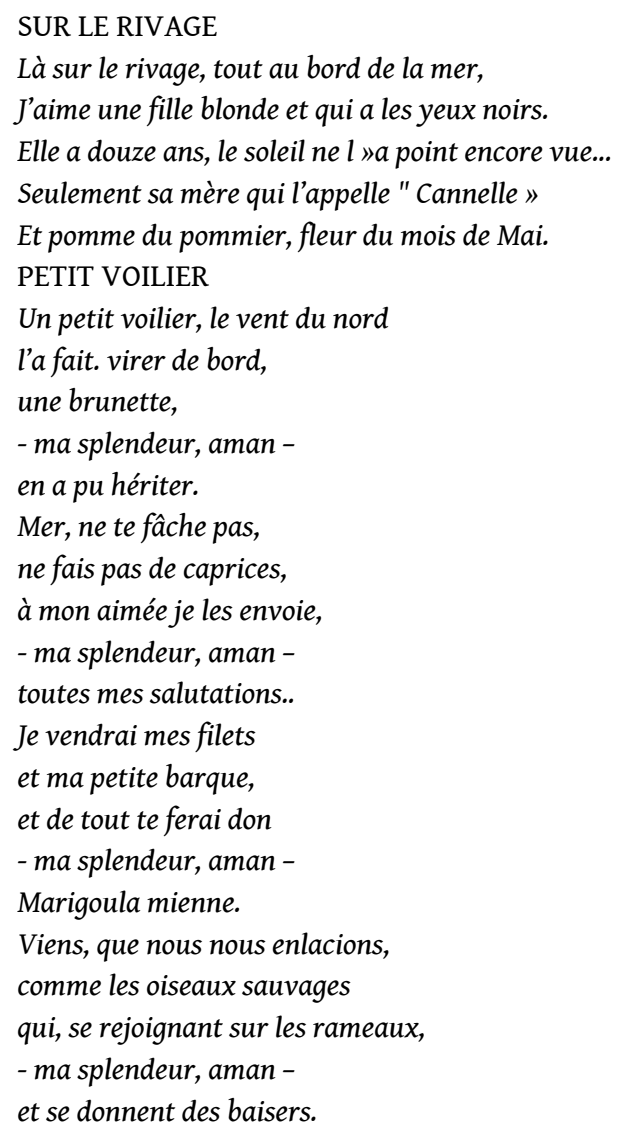

\section{Les fêtes}

Les fêtes, qui réunissent la société micrasiatique, sont, indépendamment des fêtes locales, celles de Noël, Saint Basile (1e janvier) et Carnaval. Y sont associés les "kalanda ", chansons (et danses) spécifiques, car si les deux premières sont des fêtes «intérieures" et, que l'on passe en famille, à la maison, elles sont aussi, et exclusivement pour la troisième, des fêtes "extérieures ", célébrées à l'église et dans les rues. La tradition en remonte incontestablement à l'Antiquité romaine. À l'époque protobyzantine est attestée la " danse en rond " païenne telle que la représente un basrelief de Samothrace ; elle est alors dansée dans les maisons et à l'église et symbolise la joie et la célébration de la naissance de Jésus; interdite par l'Église ensuite, n'en subsistent que les kalanda, attestés par Jean Tzetzis au XII ${ }^{e}$ siècle. Celui-ci, scholiaste et 
poète byzantin de Constantinople, est le premier à utiliser et généraliser le vers "démotique» de quinze syllabes utilisé dès lors pour la plupart des chansons populaires du monde hellénique. En Asie Mineure au début du XX siècle, les chanteurs de kalanda sont appelés "sonneurs", mais ne portent pas de déguisements et le triangle rappelle le simandre de l'ancienne danse en rond.

Les témoignages, les transcriptions orales de Phocée, Smyrne, Makri et d'autres cités ioniennes, présentent comme typique de leur «patrie » de tenir, tard dans la nuit des veilles de Noël et de la Saint Basile, des petits bateaux ou petites églises avec une lanterne de carton découpée en étoile au sommet. Petits et grands parcouraient ainsi les rues, frappaient aux portes et chantaient, attendant une obole; le corpus des kalanda d'Asie Mineure est immense ${ }^{17}$ :

Ouvrez donc vos cassettes fermées à triple tour

Et donnez pour nos peines un peu de votre argent.

Si vous êtes des seigneurs, osez les pièces d'or,

Si vous n'en êtes pas, d'argent ou des piécettes

Et si vous êtes des plus pauvres

Deux poules en paire feront l'affaire.

Donnez pour notre peine, selon votre plaisir.

Que nombreuses vous soient les années de vie,

Puissiez-vous en jouir avec santé et prospérité.

Les "Apokriatika", kalanda de Carnaval, suivent les mêmes traditions, différents seulement par les thèmes et l'utilisation d'instruments spécifiques que l'on retrouve aussi dans les autres chansons populaires, mais pas pour les kalanda de Noël : toubéléki, zilia, cuillères, verre et comboloï. Le refrain de l'un d'entre eux, d'Aïvali, reprend celui d'une célèbre cantada de Cephalonie :

Nous marchions le long du rivage

Et toujours nous parlions de toi.

Le long du rivage, en marchant sur la plage

Nous allions, tous les deux enlacés.

D'Érythrée (Cesme) provient un Apokriatiko dont la renommée s'était répandue au-delà de la localité et qui, apporté à Réthymnon par les réfugiés, se chante toujours en Crète. Il mêle tradition locale et " extérieure " puisque ici, la femme s'adresse à son mari, le "Vourliote » venu donc de Vourla, On y lit la liberté de parole et de sentiments des femmes en certaines circonstances, ici les ripailles de Carnaval.

VASSILIKOS (LE BASILIC)

Le basilic embaume ici, un Vourliote arrive,

laissez-moi le voir, car je vais rendre l'âme,

la la la, lara.

Viens que je te voie, guérisse et ne tombe pas là, morte,

que je ne tombe pas là, morte, viens que je te voie et guérisse.

Ces beaux yeux tendres, à nuls autres semblables,

sont les plus beaux de tous et de tous les plus grands,

la la la, lara...

Qu'on me laisse rester là, jour et nuit, à te regarder,

jour et nuit à te regarder, ah, si on me laissait.rester.

Quatre biens comptent en ce monde, les deux sont ta seigneurie,

les autres deux tes frères et ta noble lignée,

la la la, lara...

Viens, te dis-je, viens ne me torture plus, je vais pleurer,

ne me torture plus, viens-t-en, viens te dis-je.

Mon bey, mon beau gaillard, mon héros et mon pacha 
dans le secret de ton gile c'est mon nom qui est inscrit,

la la la, lara...

La mer cogne sur le sable, je t'aime, mais que faire,

je t'aime, mais que faire, la mer cogne sur le sable.

Le sable infini, on m'a mise à le compter

les boutons de ton gilet, à les broder d'or,

la la la, lara...

Lève-toi mon soleil et ma lumière, donne-moi ton doux baiser,

donne-moi ton doux baiser, lève-toi mon soleil et ma lumière.

Quarante-cinq coups de couteau, l'un sur l'autre,

et quarante encore je pourrai te donner, mais aucun autre ne t'aura,

la la la, lara..

Viens que je te voie et guérisse...

Ces quelques exemples de chants populaires de l'Asie Mineure égéenne que nous avons présentés ici ne rendent pas compte, même en considérant exclusivement les textes, de toute la richesse thématique du genre. Nous n'avons pu aborder les chants de noces, la berceuse, le vaste champ des chansons couvrant chacune des actions quotidiennes des femmes à la maison (broderie, cuisine, éducation des enfants...). ni la chanson politique ou mémorielle. Par ailleurs, l'amané qui est en Ionie comme dans toute l'Asie Mineure (et à Constantinople) l'une des formes d'expression les plus populaires des couches sociales défavorisées et des vicissitudes de la vie des Grecs, bien au-delà des joies, déceptions, problèmes et malheurs des petites bourgeoisies urbaines constitue à lui seul un genre. Le nombre, l'originalité, la variété tant musicale que thématique, l'intérêt linguistique, littéraire, sociétal, voire idéologique qu'il présente ne cesse de croître à la Belle Époque, cet âge d'or pourtant du développement économique et socioculturel des Micrasiates.

Quelle qu'elle soit cependant, la chanson populaire porte en elle et transmet une forme de cette culture et constitue une mémoire de l'hellénisme d'Ionie. Elle est un matériau d'étude musicologique, mais permet aussi d'approche des mentalités, des comportements, des problématiques et évolutions des "petites patries ». La mémoire, l'évocation, les images de Phocée, d'Aïvali, des quartiers de Smyrne, les rivages et la mer égéens, la vie quotidienne sont, certes, des topoi que la littérature du $\mathrm{XX}^{\mathrm{e}}$ siècle développe et analyse. Mais les chants populaires, dont nous avons vu combien l'omniprésence avait frappé, séduit les voyageurs et intellectuels européens dès le début du XIXe siècle, sont aussi témoins remarquables parce qu'ils appartiennent à tous et leur immense registre rend compte, sans artifices, d'une multitude de regards sur la langue, les moeurs la vie tant collective que privée d'une importante population.

\section{BIBLIOGRAPHIE}

Desmet-Gregoire, Hélène et François Georgeon, (2007), Cafés d'Orient revisités, Paris : Éd. CNRS.

Falbos, Philippos, (1964), « La variante smyrniote de Fiorentino », in Revue d'Art, n 117, Athènes. Georgelin Hervé, (2005), La fin de Smyrne, Paris : Éd. CNRS. 
Horbos, Nicolaos, (1998), Ancienne Phocée d'Asie Mineure (en grec) Thessalonique.

Legrand, Émile « Quatre contes grecs recueillis à Smyrne en 1875 et traduits par E. Legrand in Revue de l'Histoire des religions, T 10, no 1, Tiré à 25 exemplaires.

Metsolis, Georges, (1980), Asie Mineure, ma patrie, (en grec) Athènes.

Raffenel, Claude-Denis, (1822), Histoire des événements de la Grèce depuis les premiers troubles jusqu'à ce jour, Paris.

Thanopoulou Maria, (2006), « Du témoignage écrit à la mémoire collective », in «Identité culturelle. Littérature-Histoire-Mémoire », Athènes : EIE.

\section{NOTES}

1. La présente étude ne considérant que les seuls textes des chants, les questions musicales, les danses ne seront pas analysées, de même le contexte politique.

2. G. Metsolis, Asie Mineure, ma patrie, Athènes, 1980, N. Horbos, Ancienne Phocée d'Asie Mineure, Thessalonique, 1998.

3. « Ils » renvoie ici aux Turcs.

4. Sauf texte originel déjà en français, tous les chants ont été traduits par l'auteur de cette communication.

5. Certaines interprétations égrènent jusqu'à vint noms de lieux et chaque ville a pu s'approprier la douleur des Pergaméens (ici Phocée).

6. Mais on trouve aussi la même chanson, comme étant « de Pringhipo » (Constantinople).

7. Philippos Falbos, « La variante smyrniote de Fiorentino », in Revue d'Art, n 117, Athènes, 1964.

8. In Revue de l'Histoire des religions, $\mathrm{T} 10, \mathrm{n}^{\circ} 1$, « Quatre contes grecs recueillis à Smyrne en 1875 et traduits par E. Legrand ». Tiré à 25 exemplaires.

9. Le rythme du syrtos et le mode mineur donnent toute son intensité expressive à ce texte. La variante postérieure reprise en Grèce par les réfugiés avec une nouvelle signification est la plus connue.

10. In Histoire des événements de la Grèce depuis les premiers troubles jusqu'à ce jour, Paris, 1822 : un affrontement entre Turcs et une flottille grecque croisant entre les îles proches et la côte égéenne, provoqua le départ de certains Aîvaliotes qui se réfugient sur des îlots, les autres continuant le combat à l'entrée des Turcs dans la ville, aidés par ces Grecs, avant le pillage et la destruction d'Aïvali. (cf aussi Photis Kontoglou, Aïvali, ma patrie). Raffenel est un philhellène ayant participé à la guerre d'Indépendance, tué à Athènes en 1827 et qui avait fondé à Smyrne en 1826 le Spectateur Orienta, feuille littéraire, critique et commerciale ).

11. Cf. Maria Thanopoulou "Du témoignage écrit à la mémoire collective ", in Identité culturelle. Littérature-Histoire-Mémoire, EIE, Athènes, 2006.

12. In Sur les routes d'Asie Mineure, Éd. Trochalia, Athènes, 1990, et La Grèce, Ligue française pour la Défense des Droits de l'Hellénisme, Paris, 1908.

13. À propos du rôle des cafés dans l'Empire ottoman, de leur diversité, voir Hélène DesmetGregoire et François Georgeon, Cafés d'Orient revisités, Éd. CNRS, 2007.

14. Cf. Hervé Georgelin, La fin de Smyrne, Éd. CNRS, 2005, p. 42.

15. Archives du KE.MI.PO de Néa Ionia.

16. La version 'rébétiko', plus connue, postérieure à 1922, est différente.

17. Alors que la même tradition existait dans les îles égéennes ou à Pringhipo (Constantinople), contrairement à la Grèce continentale. 


\section{RÉSUMÉS}

Considérées comme la «mémoire" des terres ancestrales perdues, les chants sont à la fois témoignages et expression, produit des classes moyennes et inférieures urbaines grecques, nées dans une population cosmopolite, non seulement à Smyrne, mais aussi dans les autres cités de l'Asie Mineure, et dans le développement économique et culturel d'Asie Mineure des années 1880-1922. Nous pouvons lire à travers elles une société avec ses conventions, sa morale, vivante, omniprésente jusqu'en 1922, et elles transmettent une forme de l'hellénisme ionien. Elles sont un matériau pour une étude musicologique et permettent aussi d'approcher les mentalités, les comportements, les problèmes et l'évolution des "petites patries", elles présentent une évocation, des images de Smyrne, des rivages de l'Égée et de la mer, de la vie quotidienne, collective comme privée, d'une population importante.

The settlement of the population is cosmopolitan, not only in Smyrna, but also in the other Ionian cities. Regarded as a "memory" of the last fatherlands, the songs are their testimony and expression, product of the urban Greek lower middle class growing up in the micrasiatic economical and cultural development of the years 1880-1922. We can read through it a society with its conventions, morals as it is found alive, omnipresent, till 1922, and transmits a form of Ionian Hellenism. It is a material for musicology study but allows also an approach of mentalities, behaviors, problems and evolutions of the "small patries" and presents evocation, images of Smyrna, the Aegean shores and the sea, the daily life, collective as private of an important population.

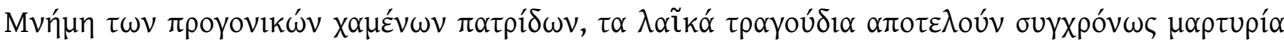

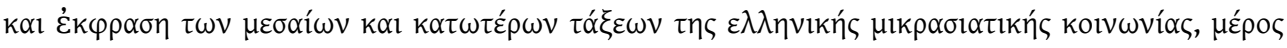

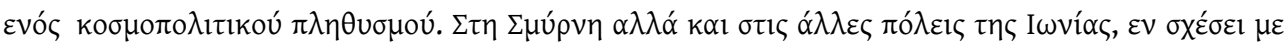

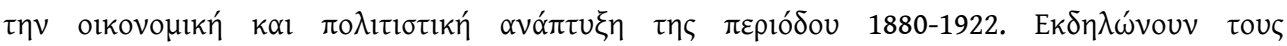

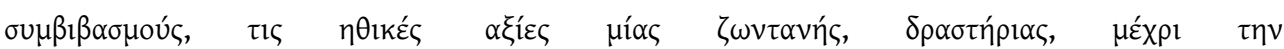

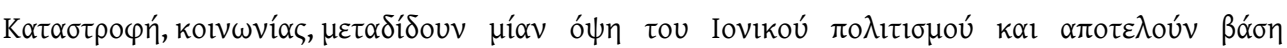

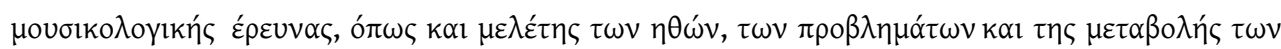

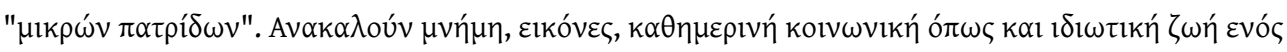

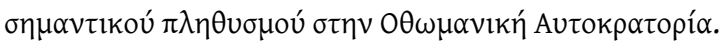

\section{INDEX}

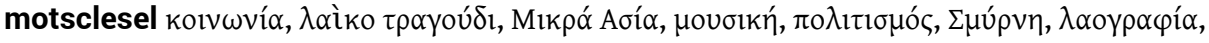

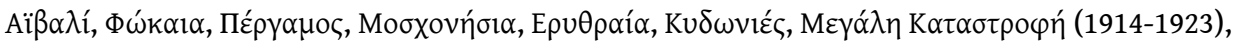

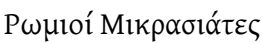

Index chronologique : dix-neuvième siècle, vingtième siècle

Thèmes : Littérature orale, Anthropologie

Index géographique : Asie Mineure, Smyrne, Ayvalik, Phocée, Pergame, Moschonissia, Cesme

Mots-clés : chants populaires grecs, Catastrophe (Grande), culture populaire, folklore

motsclestr Anadolu, İzmir, Ayvalik, Foça, Bergama, Moschonissia, Çesme, Ulusal Kurtuluş Savaşı

(1914-1923)

motsclesmk МАЛА АЗИЈА, СМИРНА

Keywords : greek folk song, folk culture, folklore, Smyrna, Ayvalik, Pergama, Phocaea, Moschonissia, Cesme, twentieth century, Orale literature 